\title{
Equilibrio e cinética de uniào de oxigênio e monóxido de carbono à hemoglobina do peixe pulmonado sul americano Lepidosiren paradoxa $\left(^{*}\right)$
}

\author{
Charles Phelps ('); Martha Farmer ( $\left.{ }^{2}\right)$; Hans J. Fyhn ( $\left.{ }^{3}\right)$; Unni E. H. Fyhn ( $)$; Rohert L. Garlick ( $\left.{ }^{(}\right)$; \\ Robert W. Noble $\left(^{5}\right)$; Dennis A. Powers ( $\left.{ }^{6}\right)$
}

\begin{abstract}
Resumo
A hemoglobina do peixe pulmonado sul americano Lepidosiren paradoxa só apresenta um componente. Os equilíbrios desta proteína respiratória foram investigados tanto no sangue como na hemoglobina purificada. Há um marcado efeito Bohr alcalino normal, assim como uma marcada sensibilidade a fosfatos orgânicos nas soluçōes de hemo. globina. Os estudos da dependência de $\mathrm{pH}$ na cinética da dissociação de oxigênio podem ser interpretados em termos de um efeito Bohr normal. A cinética de combinação de monóxido de carbono apresenta uma dependência de pH pouco usual. Estes resultados são discutidos em termos do modelo de dois estados de Monod, Wyman \& Changeux (1965).
\end{abstract}

\section{INTRODUÇÃO}

A transição de respiração aquática à respiração aérea em peixes é um passo evolutivo de significação. Peixes que apresentam tanto adaptações estruturais marcadas quanto as modificações fisiológicas nesta direção, encontram-se principalmente em águas tropicais de estuários.

O peixe pulmonado sul americano, Lepidosiren paradoxa, é o mais avançado, estruturalmente, dos peixes que respiram ar. Neste dipnoano, a bexiga de ar tem-se transformado em um pulmão pareado e septos internos dividem o espaço aéreo em compartimentos cada vez menores que terminam em lóbulos banhados por vasos sangüíneos. Se as adaptações estruturais e fisiológicas estão bem documentadas, há pouca informação disponível sobre as características moleculares de sua hemoglobina. Este trabalho se propõe a documentar as propriedades de equilíbrio de oxigênio do sangue e da hemoglobina fracionada tanto providos quanto desprovidos de fosfatos orgânicos e, além disto, descrever alguns aspectos de cinética de reação da hemoglobina com as ligações de oxigênio e monóxido de carbono.

\section{MATERIAIS E MÉTODOS}

Amostras do peixe pulmonado sul americano (Lepidosiren paradoxa) foram obtidos de um pescador local em novembro de 1976 durante uma expedição no R/V "Alpha Helix" no rio Solimões num local aproximadamente 30 $\mathrm{km}$ rio acima da união deste com o rio Negro para formar o Amazonas. A coleta foi feita no final da época seca quando o nível da água estava no ponto mais baixo nos lagos e rios. Os animais (peso corporal $370-2.000 \mathrm{~g}$ ) não estavam estivando quando coletados e foram mantidos em aquários por 2.6 dias antes de ser sangrados. O sangue foi obtido por punção cardíaca de animais, esfriados sob gelo, com uma seringa de vidro heparinizada fria $(100 \mu)$ de heparina de sódio (5.000 1. U./ml) em $\mathrm{NaCl} 1.7 \%$ por $5 \mathrm{ml}$ de sangue). As células foram centrifugadas a 1.000 r.p.m. numa centrífuga refrigerada (Sorvall Modelo RC2-B, I. Sorvall, Norwalk Conneticut, U.S.A.) durante 5 minutos. O sobrenadante foi removido e as células foram lavadas três vezes, usando o mesmo processo e suspensas em $\mathrm{NaCl} 1,7 \%$ $(\mathrm{W} / \mathrm{v})$ contendo $5 \mathrm{mM}$ de tampāo tris $\mathrm{pH} 8,0$. As células foram então lisadas por exposição a $1 \mathrm{mM}$ de tampão tris $\mathrm{pH} 8,3$ a $4^{\circ} \mathrm{C}$., durante 20 minutos. O cloreto de sódio foi agregado até uma concentração final de $100 \mathrm{mM}$ e os remanentes celulares foram retirados por centrifugação a 15.000 r.p.m.

\footnotetext{
(•) - Versão origina inglesa publicada em Comp. Blochem. Physiot. vol, 62 A (1). 1979.

(1) - Department of Biological Sclences, University of Lancaster, Lancaster LA1 4YO, Lancs, U.K.

(2) - Duke University, Marine Laboratories, Beaufort, N. C. 28516.

( 3 ) - Zoological Institute, University of Oslo, Blindern, Oslo 3, Norway.

(4) - Department of Zoology, University of Texas at Austin, Austin, Texas 78712

(5) - Dept. of Medicine \& Biochemistry, Veterans Administration Hospital, S.U.N.Y., Buffalc, New York 14215., Established Investigator of the American Heart Association.

(6) - Department of Biology, Johns Hopkins University, Baltimore, Maryand 21218.
} 
A dessalinificação foi realizada numa coluna $(110 \times 1,5 \mathrm{~cm})$ de meio G-25 (Pharmacia Fine Chemicals, Uppsala Suecia) equilibrada em tris $1 \mathrm{mM} \mathrm{pH} \mathrm{8,3.} \mathrm{O} \mathrm{fracionamento} \mathrm{de} \mathrm{so-}$ luções de hemoglobina foi realizada numa coluna $(15 \times 2,5 \mathrm{~cm})$ de resina de camada mista AG 501-x8(D) do Bio Rad Laboratories, Richmond California, acima da qual foram colocadas 2 camadas de intercambiadores iônicos Dowex - 50W - $\mathrm{NH}_{4}+$ e Dowex 1 - Acetato (Dow Chemical Co. Michigan, USA). Mantida velocidade de Fluxo de $1 \mathrm{ml} / \mathrm{min}$.

As eletroforeses em gel de disco com o $\mathrm{pH} 8.9$ foram realizadas como descrito por Fyhn et. al. (1978). Dos mesmos geis após coloração, foi calculado a relaçäo entre distância de migração do componente da hemoglobina e a de albumina de sôro bovino e usado para efetuar comparações de componentes de hemoglobina em geis de diferentes corridas. Estas taxas são chamadas mobilidades relativas.

As medições de equilíbrio de oxigênio foram realizadas usando-se tonômetros de vidro como o descrito por Allen et al., 1950 e Riggs \& Wolbach (1956). Os resultados foram calculados como os descritos por Nagel et al. (1965). Todos os experimentos foram realizados a $20^{\circ} \mathrm{C}$ com as concentrações de hemoglobina aproximadamente entre $50-100 \mu \mathrm{M}$ em hemo-equivalentes. A oxidação da solução previamente desoxigenada foi observada espectrofotometricamente e duas longitudes de onda, 560 e $576 \mathrm{~nm}$. As adições de gás foram, de ar ou de oxigênio puro. O tempo de equilíbrio após cada adição variou entre $10-20 \mathrm{~min}$. Os tampões usados foram todos com $\mathrm{I}=0,05$, com Bis Tris, na amplitude de $\mathrm{pH}$ de $5,25-7,3$ e Tris para valores de $\mathrm{pH}$ acima de 7,3.

A formação de metahemoglobina foi medida como descrito por Benesch et al. (1965) e sempre foi menor que $3 \%$. Mediçōes de equilíbrio de oxigênio no sangue completo foram feitas utilizando o aparelho Hem-O-Scan (American Instrument Co., Silver Springes, Md.) descrito por Powers et al. (1978) .

As medições cinéticas foram feitas a $20^{\circ} \mathrm{C}$, com um aparelho de mistura rápida de fluxo fechado similar ao descrito por Gibson e Milnes (1969). Soluções de hemoglobina idênti. cas em composição foram as usadas para medições de equilibrio.

As dissociações cinéticas de oxigênio foram medidas pelo processo de variação de $\mathrm{pH}$ como o descrito por Noble et al. (1970). Hemoglobina oxigenada em Tris $1 \mathrm{mM}, \mathrm{pH} 8,0$ foi misturada com uma solução de ditionito em um tampäo de $\mathrm{pH}$ desejado, $\mathrm{I}=0,05$. A concentração final da hemoglobina foi aproximadamente $30 \mu \mathrm{M}$ em hemoequivalentes e a reação fơ seguida tanto 560 quanto $540 \mathrm{~nm}$, independentemente.

As combinaçöes cinéticas de monóxido de carbono com hemoglobina desoxigenadas foram medidas misturando-se soluçōes de hemoglobina desoxigenadas em tampões do $\mathrm{pH}$ desejado de força iônica I $=0,1$, contendo uma concentração conhecida aproximadamente 87 $\mu \mathrm{M}$, de monóxido de carbono dissolvido em água. Após misturar a concentração de hemoglobina foi de c.a. $3 \mu \mathrm{M}$ em hemoequivalentes. A reação foi seguida a 420 a $435 \mathrm{~nm}$, independentemente.

\section{RESUltados E DISCUSSÃo}

Os estudos no sangue completo do animal revelam que a hemoglobina está metade saturada a $8,3 \mathrm{~mm} \mathrm{Hg} \mathrm{pO}$ a $30^{\circ}$ e $\mathrm{pH} 8,06$. Ao reequilibrar a amostra de sangue em presença de $5,6 \% \mathrm{v} / \mathrm{v}$ de $\mathrm{CO}_{2}$, o $\mathrm{pH}$ da amostra caiu a

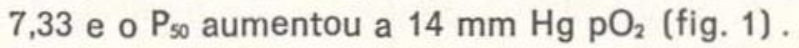
Assim, a $30^{\circ} \mathrm{C}$ há evidência de um efeito Bohr significativo que pode ser composto de dois efeitos $\mathrm{H}+$ e $\mathrm{CO}_{2}$, com magnitude aparente. entre $\mathrm{pH} 7,33-8,06$ de :

$$
\frac{\Delta \log \mathrm{P}_{50}}{\Delta \mathrm{pH}}=-0,31
$$

Foi realizada uma exaustiva investigaçăo do equilíbrio de oxigênio em hemoglobina isolada. A eletroforese em gel poliacrilamida (Fyhn et. al. 1978) revelou só um componente. A hemoglobina apresenta uma mobilidade de $0,66 \pm 0,01$ com relação à albumina de soro bovino, e a Figura 2 compara os padrões eletroforéticos obtidos de hemolisados de Lepidosiren paradoxa com os do homem.

A dependência de $\mathrm{pH}$ da curva de dissa ciação de oxigênio foi medida para material que tinha sido liberado de fosfatos orgânicos 


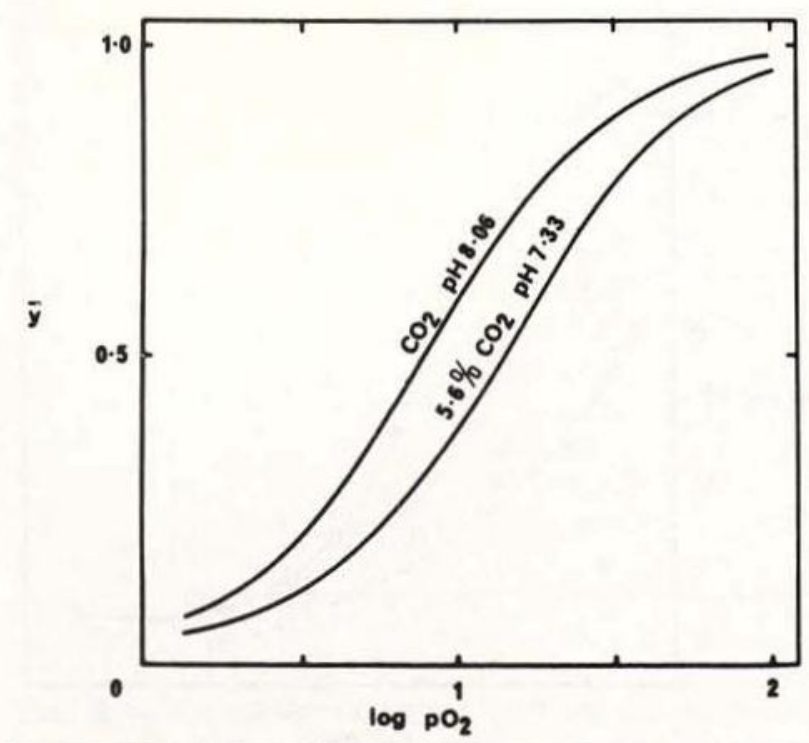

Fig. 1-Curvas de equilíbrio de oxigênio do san. gue completo de Lepidosiren paradoxa sob as condições especificadas. A ordenada indica saturação fracional da hemoglobina pelo oxigênio e a abscissa $\circ$ valor de $\log \mathrm{pO}_{2}$ expresso em $\mathrm{mm} \mathrm{Hg}$.

e para soluções idênticas às quais fơi adicionado ATP até uma concentração final de $1 \mathrm{mM}$ (Fig. 3). As duas curvas são largamente similares sendo que a presença de fosfato orgânico tem diminuído grandemente a afinidade da hemoglobina, como é de esperar-se se estabilizou à conformação deóxi.

A magnitude do efeito Bohr é muito maior em presença de ATP $1 \mathrm{mM}$, e sua resposta a $\mathrm{pH}$ entre 7,00 a 8,00 é cinco vezes maior em presença de fosfato orgânico (Fig. 3) .

Os dados de equilíbrio refletem o comportamento de uma hemoglobina com respostas não muito diferentes as da hemoglobina humana A. A magnitude do efeito Bohr em hemoglobina $\mathrm{A}$ a $25^{\circ} \mathrm{em}$ sistemas tampão similares é de $-0,30$ entre $\mathrm{pH} 6,8$ e 8,8 e o efeito de fosfato orgânico em excesso diminui a afinidade de oxigênio por um fator de 10 (Imai, 1974). Parece claro que quaisquer adaptações moleculares necessárias no transcurso da evolução da água para o ar, já estão completas ao nível de evolução do peixe pulmonado.

A dependência de $\mathrm{pH}$ na velocidade de dissociação de oxigênio da hemoglobina de Lepid̋osiren é apresentada tanto em presença quanto em ausência de $1 \mathrm{mM}$ ATP. A dependência de $\mathrm{pH}$ observada é indicativa de um efeito Bohr normal que é aumentado apreciavelmente por ATP. Na ausência de ATP, $k$ aumenta quase 3 vezes à medida que o pH é baixado entre 8 e 9 a 6 . Na presença de ATP, esta mudança é de quase 10 vezes. As velocidades de reação absolutas não são surpreendentes, estando mais ou menos na mesma faixa das reportadas para hemoglobina de mamíferos (Fig. 4).

A dependência de $\mathrm{pH}$ da reação de monóxido de carbono com hemoglobinas desoxigenadas de Lepidosiren também é apresentada,

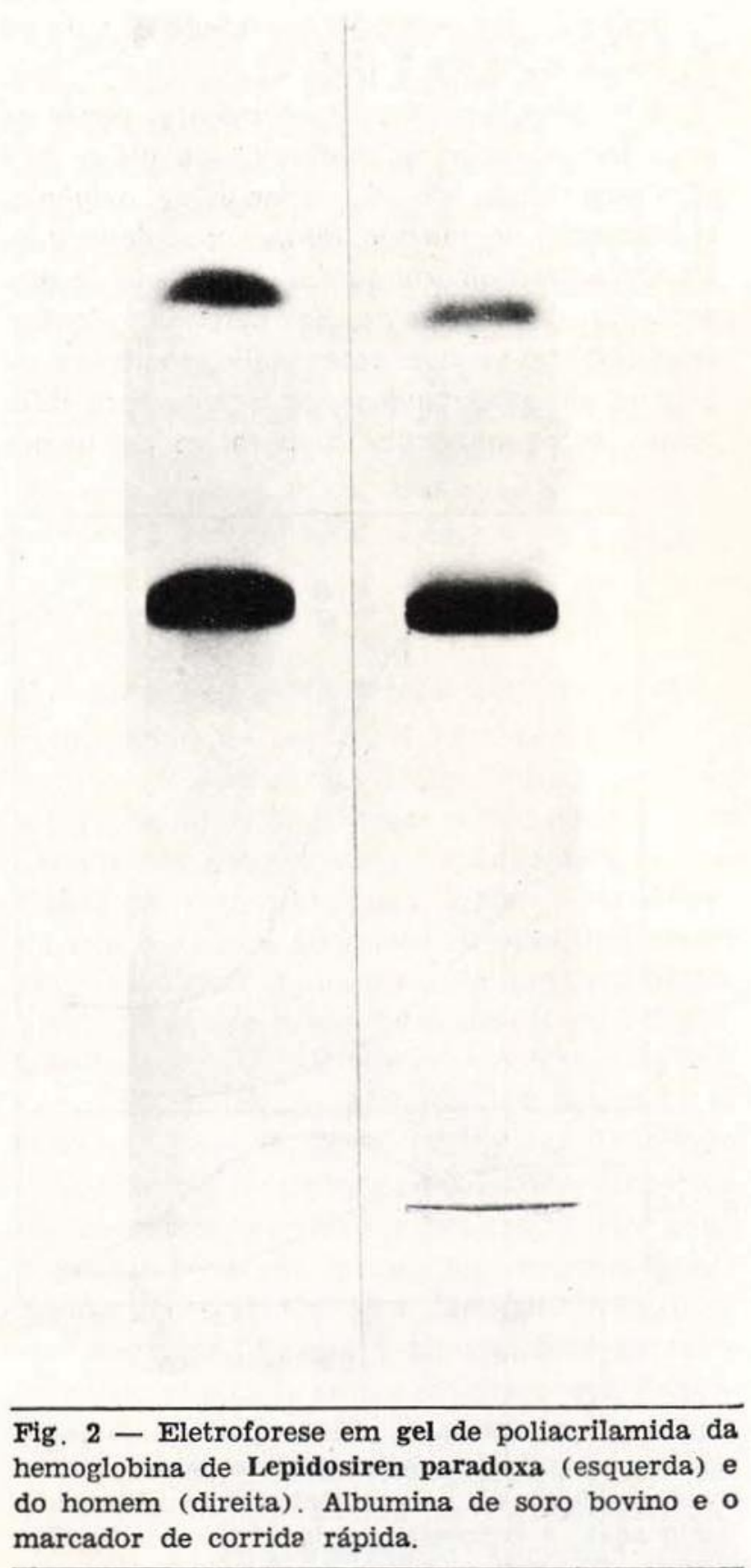


na presença e ausência de $1 \mathrm{mM}$ ATP. Esta dependência de $\mathrm{pH}$ é muito estranha. $\mathrm{Na}$ ausência de ATP, esta dependência tem duas fases bem marcadas. Inicialmente a velocidade de reação aumenta com o aumento de $\mathrm{pH}$, mais a um $\mathrm{pH}$ de aproximadamente 7,5 , a dependência é reversa e a velocidade diminui abruptamente com maiores aumentos no $\mathrm{pH}$. O ATP modula esta dependência e tem efeito significativo nesta reação a valores de $\mathrm{pH}$ muito altos. Só a pH 9,0 é insignificante o efeito. Assim podemos concluir que há união preferencial de ATP ao estado estrutural deóxi desta molécula de hemoglobina ainda a valores de $\mathrm{pH}$ de entre 8 e 9 (Fig. 5) .

Em princípio, é surpreendente observar uma dependência tão ordinária de $\mathrm{pH}$ e ATP para velocidade de dissociação de oxigênio, encontrando ao mesmo tempo uma dependência tão extraordinária para a velocidade de dissociação de monóxido de carbơno. Porém, deve lembrar-se que estes dois processos cinéticos são controlados por fatores bem diferentes numa molécula cooperativa de hemo-

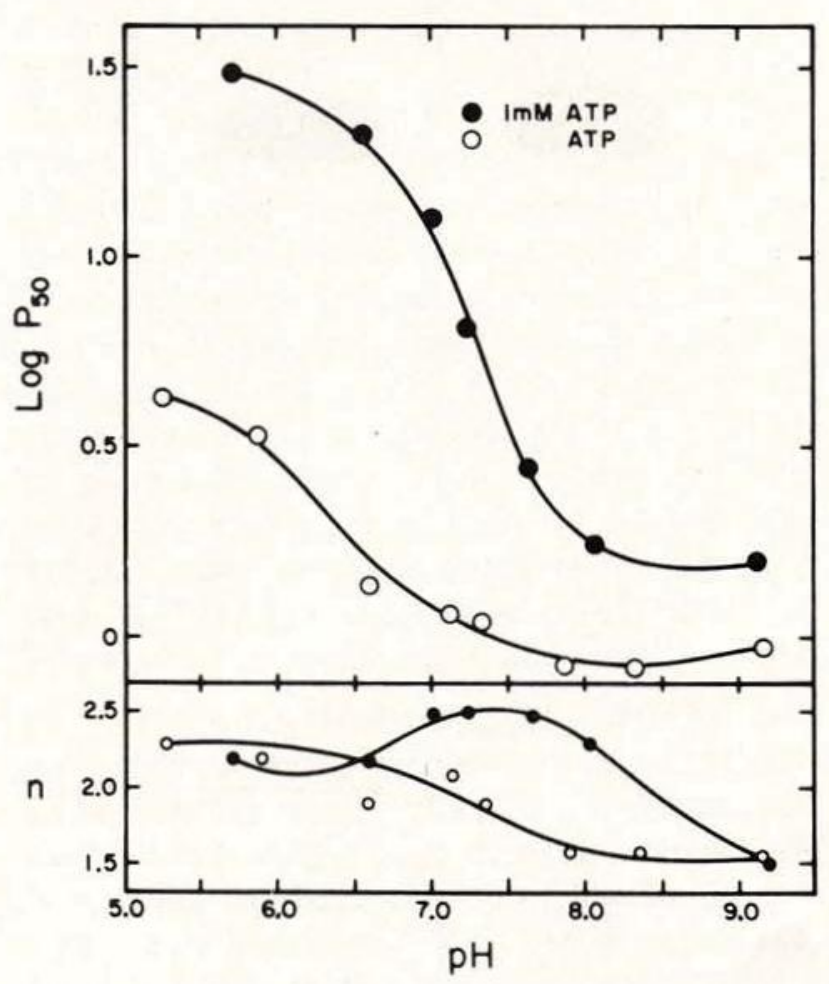

Fig. 3 - Dependência de pH de equilíbrio de oxigênio com hemfoglobina de Lepidosisen paradoxa mostrando o efeito de preparações fracionadas e não fracionadas. A concentração de ATP foi de $1 \mathrm{mM}$.

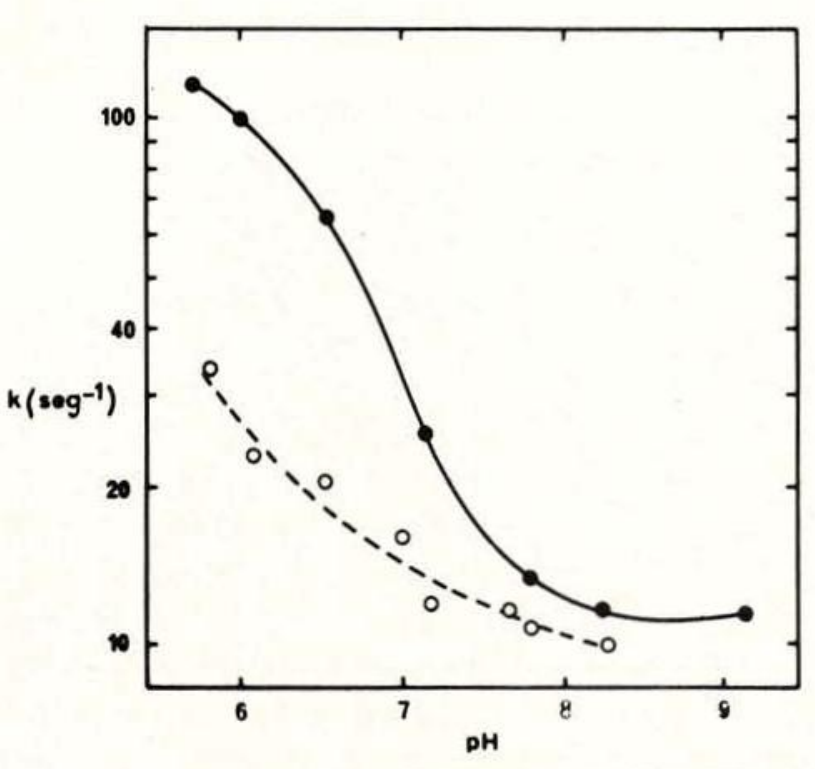

Fig. 4 - Dependência de pH na constante da velocidade de dissociação de oxigênio de oxihemoglobina de Lepidosiren paradoxa, indicando o efeito de preparação fracionada e não fracionada. A concentração de ATP foi de $1 \mathrm{mM}$, temperatura $20^{\circ} \mathrm{C}$.

globina. Isto pode ser interpretado em base a um modelo alostérico de dois estados (Monod et al., 1965). Isto propõe que subunidades podem unir-se com duas estruturas quaternárias diferentes, porém, equilibradas. As diferentes ligações entre subunidades nestas duas moléculas, alteram a conformação individual da subunidade de modo que em uma estrutura, chamada $R$, a afinidade das subunidades para ligandos é maior que no outro estado, T. A ligação do ligante inevitavelmente leva o equilíbrio em direção ao estado $R$, resultando em aumentos sucessivos de afinidade à medida que aquela se processa. Outro postulado neste esquema é que dentro de cada estrutura quartenária a afinidade de uma subunidade não é influenciada pelo estado de ligação de suas vizinhas. Assim com os experimentos aqui apresentados, o perfil cinético de dissociaçăo de oxigênio quando está sendo influenciado pelo estágio no processo de desoxigenação no qual a proteína passa do estado $R$ a $T$, é limitado na velocidade, primariamente pela dissociação de oxigênio do estado $R$ de alta afinidade da molécula de hemoglobina. Em contraste com isto, a velocidade de combinação de $\mathrm{CO}$ reflete primariamente o comportamento funcional do estado $\mathrm{T}$ ou deóxi da proteína. 


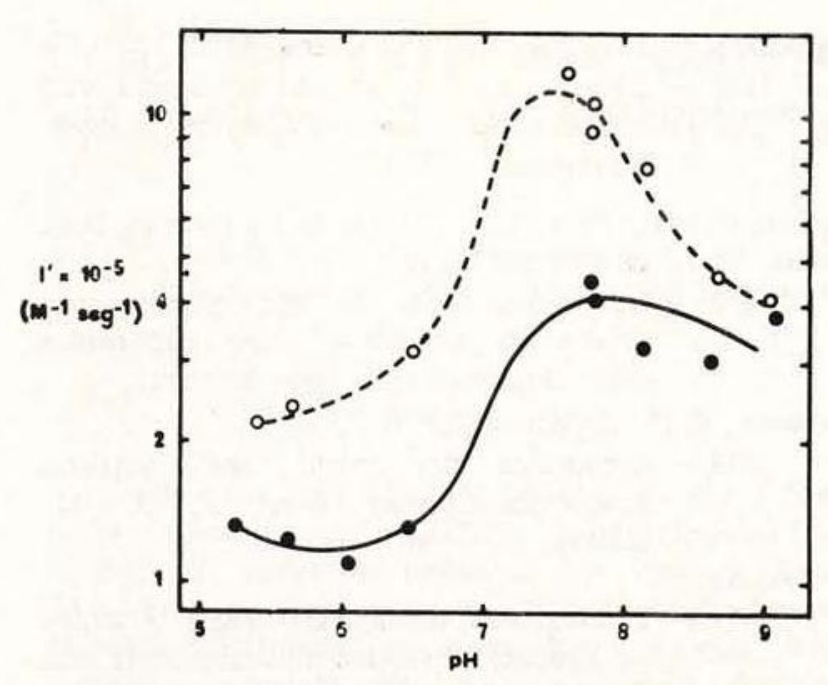

Fig. 5-Dependência de pH entre a constante de velocidade de combinação entre monóxido de carbono e desoxihemoglobina de Lepidosiren paradoxa. Os dois traços indicam os parâmetros para hemoglobinas fracionadas e não fracionadas de ATP.

A dependência de $\mathrm{pH}$ das constantes de velocidade sugere que as propriedades funcionais do derivado desoxigenado da hemoglobina de Lepidosiren são sensitivas ao estado de ionização de um número de radicais ácidos na hemoglobina.

\section{DISCUSSÃo GERAL}

O pulmão dipnoano desenvolveu-se como um órgão de emergência nos peixes pulmonados africanos e sul americanos (Protopteros e Lepidosiren, respectivamente), porém, ventilação pulmonar é atualmente obrigatória, sendo que as brânquias só têm função vestigial, funcionando como osmoreguladores. $O$ peixe pulmonado australiano Neoceratodus não é um respirador de ar obrigatório e morre fora d'água.

Um fato curioso do tipo de vida de Lepidosiren que contradiz sua obrigada aerobiose é realizada pelo macho. Durante a época em que cuida dos ovos, o macho não emerge à superfície e desenvolve filamentos respiratórios consideráveis nas aletas pélvicas. Estes são primeiramente observáveis após o aparecimento e atrofiam pouco após a eclosão dos ovos quando as guelras larvais degeneram e os jovens começam a respirar ar (Krogh, 1941).
Johansen \& Lenfant (1968) e Lenfant \& Johansen (1968) estudaram as adaptaçōes fisiológicas envolvidas na transição para respiração aérea em peixes pulmonados. Tanto as propriedades da hemoglobina quanto o comportamento respiratório sugerem que Neoceradotus é um respirador branquial e que os pulmões só servem uma função auxiliar quando o $\mathrm{pO}_{2}$ externo é baixo. Porém, os Lepidosiren e os Protopterus usam seus pulmões de maneira funcional, ainda que utilizem suas guelras vestigiais para grande parte da eliminação de dióxido de carbono.

Seria muito bom poder generalizar os fatores observados na transição de respiração aquática e aérea, mas isto não parece possível. Com base em estudos feitos sobre a sensibilidade a dióxido de carbono entre os peixes teleósteos têm-se sugerido que peixes de movimentos lentos que habitam águas estagnadas tendem a mostrar efeitos Bohr menores sendo que espécies ativas de água em movimento rápido bem oxigenado apresentam respostas maiores em afinidade de oxigênio com mudanças menores em $\mathrm{PCO}_{2}$. Um exemplo deste últimn masn é observado na cavala onde o efeito $\Delta \log P_{50}$

Bohr - $=-1,2$ (Prosser, 1961). $\Delta \mathrm{pH}$

Os valores deste efeito reportados para peixes pulmonados por Lenfant \& Johansen (1968) fo ram: - Neoceratodus -0,62; Protopterus - 0,47 e Lepidosiren $-0,24$. O valor obtido por nós para Lepidosiren é claramente dependente da quantidade de fosfato orgânico ligado. Assim, sendo que o sangue completo resultou um efeito Bohr de $-0,31$, a figura 3 revela que para hemoglobina fracionada em faixa de $\mathrm{pH}$ de $7,0-8,0$, o valor pode ser tão baixo como $-0,18$ e na presença de $1 \mathrm{mM}$ de ATP o valor aumenta até $-1,05$. Oldham \& Riggs (1969) reportam que o equilíbrio de oxigênio para as duas espécies de peixes pulmonados africanos é diferente, tanto em afinidade quanto em resposta Bohr. Assim, para Protopterus aethiopicus capturados em lagoas fundas, a hemoglobina apresenta maior afinidade com o oxigênio que Protopterus annectans obtido de pântanos. As duas espécies apresentam hemoglobinas de componentes múltiplos, equilíbrios de oxigênio sigmóides e cooperatividade expressa como 
valores $\mathrm{n}$ que são dependentes do $\mathrm{pH}$. A hemoglobina de Protopterus aethipicus tem um efeito Bohr de -0,8 sendo que o de Protopterus annectans resultou valores de $-0,54$. Não é fácil apoiar a sugestão de efeito Bohr reduzido em águas estagnadas, o que é também mostrado em outro grupo animal, os anfíbios, estudados por Lenfant \& Johansen (1967). Neste grupo, a tendência é reversa. Necturus apresenta um efeito Bohr de -0,13, Amphiuma -0,21 e Rana catesbeiana -0,29.

\section{AGRADECIMENTOS}

Este trabalho foi apoiado pela Bolsa PCM06451 para estudo a bordo do R/V "Alpha Helix". Agradecemos aos brasileiros pela sua ajuda e por ter possibilitado a entrada do "Alpha Helix" no Rio Solimões. Queremos também, agradecer ao Capitão Clarke e tripulação pela cooperação. Apoio adicional foi dado pelo Conselho de Pesquisas para Ciência e Humanidade da Noruega (H.J. e V.E.H.F.), Bolsa do NSF DEB - 76 - 19877 (D.A.P.), Bolsa do NIH HL -15460 (a J. Bonaventura para apoio de M.F.), Bolsa NSF PCM (a A. Riggs), a National Geographic Society (D. A. P.) e Bolsa NIH GM 21314 (a A. Riggs). M. Farmer percebe a Bolsa de Pesquisas $n^{\circ}$ 303-3765 da Duke University.

\section{SUMMARY}

The haemoglobin of the South American lungfish Lepidosiren paradoxa has a single component. The equilibria of this respiratory protein with oxygen have been investigated both in the blood and with the purified haemoglobin. There is a substantial, normal, alkaline Bohr effect and marked sensitivity to organic phosphates in the haemoglobin solutions. Studies on the $\mathrm{pH}$ dependence of the kinetics of oxygen dissociation can be interpreted in terms of a normal Bohr effect. The kinetics of combination of carbon monoxide have an unusual $\mathrm{pH}$ dependence. These findings are discussed in terms of the two state model of Monod, Wyman and Changeux (1965).

\section{BIBLIOGRAFIA}

Allen, D.W ; GUTHE, K.F. \& WyMAN, J.

1950 - Further studies on the oxygen equilibrium of hemoglobin. J. Biol. Chem. 187:393-410
BeNESCH, R.; MACDUfF, G. \& BenesCH, R.E.

1965 - Determination of oxygen equilibria with a versatile new tonometer. Anal. Biochem., 11:81-87.

FYHN, U.EH.; FYHN, H.J.; DAvIS, B.J.; POWERS, D.A.; FINK, W.L. \& GARLICK, R.L.

1978 - Heterogeneidade de hemoglobina nos peixes da Amazônia. Acta Amazonica 8(4) : Suplemento. (Este volume),

GIBSON, Q.H. \& MILNES, L.

1964 - Apparatus for rapid and sensitive spectrophotometry Biochem. J., 91: 161-171.

IMAI, $\mathbf{K}$.

1974 - Hemoglobin chesapeake (92a, Arginine -, Leucine) Precise measurements and analyses of oxygen equilibrium. J. Biol. Chem., 249:7607-7612.

Johansen, $\mathrm{K}$. \& Lenfant, C.

1968 - Respiration in the African lungfish II control of breathing. J. Exp. Biol., 49: 453-468.

KROGH, A.

1941 - The comparative Physiology of respiratory Mechanisms. Philadelphia, Univ. of Pennylvania.

LENFANT, C. \& JohnNSEN, K.

1967 - Respiratory adaptations in selected amphibians. Respiration Physiol., 2: 247-260.

1968 - Respiration in the African lungfish Protopterus acthiopicus I Respiratory properties of blood and normal patterns of breathing and gas exchange. J. Exp. Biol., 49:437-452.

Monod, J.; Wyman, J. \& Changeux, J.P.

1965 - On the Nature of Allosteric Transitions: A Plausible Model. J. Mol. Biol., 12: 88-118.

NAgel, R.L.; WITTENBerg, J.B. \& RANNEY, H.M.

1965 - Oxygen equilibria of the hemoglobin haptoglobin complex. Biochim. Biophys. Acta., 100:286-289.

Noble, R.W.; PARKhURST, L.J. \& Gibson, Q.H.

1970 - The effect of $\mathrm{pH}$ on the reactions of oxygen and Carbon Monoxide with the hemoglobin of the carp, Cyprinus carpio. J. Biol. Chem., 245:6628-6633.

Oldham, J. \& Riggs, A. apud Riggs, A.

1969 - Properties of fish hemoglobins. Chapt. 6:245-246. Fish Physiology, v. 4, 1970.

RIgGS, A. \& Wolbach, R.A.

1956 - Sulfhydryl. Groups and the-structure of hemoglobin. J. Gen. Physiol., 39: 585-605. 